\title{
P01-2-29
}

Poster session

\section{Molecular characterization of cardioprotective exosomes secreted from postnatal cardiac Nkx2.5+ cells}

\section{Wen-Pin Chen, Wei-Ping Liang, Min-Yi You, You-Yi Li, Po-Chiang Hsiao, Szu-Ying Chen, Cheng- Pu Huang}

Institute of Pharmacology, National Taiwan University, Taiwan

Background: A83-01, a TGFb\&\#61538;RI inhibitor, could prominently increase cardiomyocyte survival in post-injured adult hearts via expanding cardiac Nkx2.5+ cells to produce the paracrine benefit in vivo. The present study aimed to characterize the molecular profile of the exosomes secreted from cardiac fibroblast [Exo(cfb)] and Nkx2.5+ cells treated with DMSO or A83-01 [Exo $(\mathrm{Nkx}+\mathrm{D})$ or $\operatorname{Exo}(\mathrm{Nkx}+\mathrm{A})]$, and to compare the functional impact of the different exosomes on adult cardiomyocyte viability in vitro.

Methods: Cardiac Nkx2.5 + cells expressing GFP driven by Nkx2.5-enhancer were enzymatically isolated from mice hearts, and were further purified by flow cytometric sorter under the exclusion of hematopoietic lineages, dead cells, and sca1+ cells. Nkx2.5+ cell-secreted exosomes were extracted from culture medium by ExoQuick-TC (System Biosciences). Exosomal proteins were identified by LC-MS/MS, and exosomal miRNAs by mouse miRNA microarray (MRmiOA7.0). Results: The molecular profiles of Exo $(\mathrm{Nkx}+\mathrm{D})$ and $\mathrm{Exo}(\mathrm{Nkx}+\mathrm{A})$ were markedly different from those of Exo $(\mathrm{cfb})$. Though ten common proteins, including thrombospondin-4, were present in Exo(Nkx+D), Exo(Nkx+A) and Exo(cfb), six proteins were exclusively in $\operatorname{Exo}(\mathrm{Nkx}+\mathrm{D})$ and seven proteins exclusively in Exo $(\mathrm{Nkx}+\mathrm{A})$. There were 30 common miRNAs that were highly expressing in Exo $(\mathrm{Nkx}+\mathrm{D})$ and Exo $(\mathrm{Nkx}+\mathrm{A})$. Either Exo $(\mathrm{Nkx}+\mathrm{D})$ or $\operatorname{Exo}(\mathrm{Nkx}+\mathrm{A})$ was better than Exo(cfb) in the enhancement of adult cardiomyocyte viability after treatment for 3 days in vitro.

Conclusions: The unique molecular profiles of $\operatorname{Exo}(\mathrm{Nkx}+\mathrm{D})$ and $\mathrm{Exo}(\mathrm{Nkx}+\mathrm{A})$ were associated with their better prosurvival effects in adult cardiomyocytes as compared to those of Exo(cfb). Stimulation or administration of cardioprotective exosomes secreted from cardiac Nkx2.5+ cells should be promising strategy to preserve post-injured myocardium. 